Meningitis is an inflammation of the pia and arachnoid maters (leptomeningitis) or the dura mater (pachymeningitis). Magnetic resonance imaging (MRI) in meningitis, as with most other forms of intracranial inflammatory or infectious diseases, is a powerful though largely non-specific diagnostic tool. The technique is used to detect the presence of disease, and also complications related to the primary process, as well as assess for alternative diagnoses. The actual diagnosis of meningitis remains largely a clinical and laboratory one. For imaging of these patients the Basic Protocol presented previously in UNIT A4.1 is utilized, to include gadolinium-enhanced sequences. An optional sequence, diffusion MRI (dMRI), is outlined, which can be employed if specific clinical situations exist to be further clarified. The parameters given here are derived from experience at $1.5 \mathrm{~T}$ and may need to be altered slightly depending on the field strength available and the specific equipment manufacturer.

\section{IMAGING OF MENINGITIS}

Presented here is the authors' routine MR head examination for inflammatory disease, with gadolinium contrast agent sequences. The total number of sequences presented is seven (scout, $T_{1}$-weighted sagittal and transverse, fluid-attenuated inversion recover (FLAIR) and fast spin-echo (FSE) $T_{2}$-weighted transverse, and post-contrast $T_{1}$-weighted transverse and coronal). This examination may be shortened considerably by limiting the use of two planes for post-contrast imaging to a single transverse plane for exams with a lower clinical suspicion for disease, or if review of the images obtained through the transverse gadolinium-enhanced images proves unremarkable. The sequences described herein are based on the authors' experience with a Marconi Medical Systems 1.5T scanner, but are expected to be equally applicable to machines from other manufacturers. The same basic scanning protocol to image for meningitis as that for cerebral abscess (UNIT A4.1) is utilized, which follows below.

Table A4.2.1 lists the hardware necessary to perform the examination, along with appropriate parameters.

NOTE: Be sure that technologists and nurses have immediate access to any emergency equipment that may be relevant to a given study, or that may be needed for a particular patient, such as a crash cart or oxygen. Also ensure only magnetic field compatible oxygen tanks are utilized if wall-source oxygen is unavailable.

\section{Materials}

Extravascular contrast agent (e.g., $0.1 \mathrm{mmol} / \mathrm{kg}$ patient body weight of gadolinium chelate from Mangevist, Omniscan, or Prohance)

Normal saline $(0.9 \% \mathrm{NaCl})$ sterile

Table A4.2.1 Equipment Parameters for Contrast-Enhanced Head Imaging Sequences

\begin{tabular}{ll}
\hline Coil type & Head \\
Gradient coil strength & $25 \mathrm{mT} / \mathrm{m}$ (or whatever the system permits) \\
Gating (cardiac, respiratory, peripheral) & No \\
Respirator or oxygen & If required by patient \\
Motion cushions & Recommended \\
Contrast agents & Yes \\
\hline
\end{tabular}

Contributed by Andrew E. Auber and Clifford Belden

Current Protocols in Magnetic Resonance Imaging (2001) A4.2.1-A4.2.13

Copyright $\odot 2001$ by John Wiley \& Sons, Inc.
BASIC

PROTOCOL

Infectious

Diseases of the Brain

A4.2.1

Supplement 1 


\section{Set up patient and equipment}

1. Interview (screen) the patient to ensure that he/she has no contraindication for MR scanning such as cardiac pacemaker or other implants containing ferromagnetic materials. Also be sure to find out if the patient has any health conditions that may require the presence of special emergency equipment during the scanning procedure, or that necessitate other special precautions. Screening forms are often employed for all patients before scanning in an MRI system.

Generally standard screening forms are used for all patients scanned in a magnetic resonance system.

The presence of any ferromagnetic metals may be a health hazard to the patient when he or she is inside the magnet, and will also affect the imaging. If in doubt as to the exact composition of the items, it is best to exclude patients with any metal implants; see Shellock (1996) for discussion of what implants may be safely scanned using magnetic resonance.

Patients may be accompanied into the magnet room by a friend or family member, who can sit in the room during the scan and comfort the patient as needed. This companion must be screened as well to ensure the absence of loose metal objects on the body or clothing.

2. If the procedure is a research protocol, have the patient sign any necessary consent form.

3. Have the patient remove all jewelry and change into a gown to eliminate any metal that might be found in clothing.

4. Have the patient remove any mascara or other metal-containing makeup to avoid local tissue heating and image artifacts.

5. Inform the patient about what will occur during the procedure, what he/she will experience while in the magnet, and how to behave, to include the following:

a. If earphones/headphones are used to protect the ears from loud sounds produced by the scanner, the patient will be asked to wear these, but will be able to communicate with the technologist at any/all times during the procedure.

b. The patient will be given a safety squeeze-bulb or similar equipment to request assistance at any time (demonstrate how this equipment works).

c. For optimum results the patient should not talk, and should avoid/minimize swallowing or other movement during each scan, i.e., as long as the banging sounds continue. Between scans, talking and swallowing are allowed in most cases, but should be avoided when comparative positional studies are being performed. The patient will be informed in these instances.

d. Nevertheless, the patient may call out at any time if he/she feels it necessary.

6. Have the patient lay supine on the scanner table. Either before or directly after the patient is positioned on the table, set up any triggering devices or other monitoring equipment necessary.

7. Establish an i.v. line through which the contrast agent can be injected, and attach this line securely to the patient so that movement into or out of the magnet will not pull at the patient's arm. It is preferable to insert the line prior to imaging. This allows no intervening motion between those scans performed before and those run after the contrast agent injection.

8. Center the patient in the head coil. Make sure that the head and neck are constrained to prevent unnecessary motion, especially if high-resolution scans are to be run. 
9. If needed, place a pillow or other support under the knees to make the patient more comfortable.

10. Use the centering light to center on the nasion of the patient and place them into the center of the magnet.

Once this step has been performed, so long as the patient does not move on the table, the table itself can be moved and then replaced in the same position as before without jeopardizing the positioning of one scan relative to another.

11. If the patient is unable to hold still, either provide an appropriate sedative, or arrange with anesthesiology for conscious or general anesthesia.

Alternatively, a low-field open magnet may be sought for scanning the claustrophobic patient. There are also fast scanning techniques that may be employed for imaging of these patients (see Chapter A5).

12. This Basic Protocol can be performed in $<30 \mathrm{~min}$.

\section{Sequence 1: Rapid three-plane pilot}

13. Run the three-plane pilot (Table A4.2.2) sequence to evaluate the patient positioning in the magnet.

14. This sequence runs in $<10 \mathrm{sec}$ and is used to position the remainder of the sequences. It is particularly useful to correct off-axis positioning in the coronal plane.

\section{Sequence 2: $T_{1}$-sagittal head}

15. This sequence serves as a true $T_{1}$-weighted sagittal study of the head. Bring the sequence for a sagittal $T_{1}$-weighted scan up onto the console and utilize the parameters in Table A4.2.3.

16. Use the pilot sequence to set up the scan levels.

Table A4.2.2 Primary Clinical Imaging Parameters for Sequence 1: Rapid Three Plane Pilot

\begin{tabular}{ll}
\hline Patient position & Supine \\
Scan type & Gradient echo \\
Imaging plane (orientation) & Sagittal, transverse, and coronal \\
Central slice or volume center & Midline head \\
Echo time $\left(T_{\mathrm{E}}\right)$ & $3.7 \mathrm{msec}$ \\
Repeat time $\left(T_{\mathrm{R}}\right)$ & $16 \mathrm{msec}$ \\
Flip angle $(\mathrm{FA})$ & $20^{\circ}$ \\
Fields of view (FOV, $\left.\mathrm{FOV}_{\mathrm{y}}\right)$ & $300 \mathrm{~mm}, 300 \mathrm{~mm}$ \\
Resolution $(\Delta x, \Delta y)$ & $2.34 \mathrm{~mm}, 2.34 \mathrm{~mm}$ \\
Number of data points collected $\left(N_{\mathrm{x}}, N_{\mathrm{y}}\right)$ & 128,128 \\
Display matrix $\left(D_{\mathrm{x}}, D_{\mathrm{y}}\right)$ & 256,256 \\
Slice thickness $(\Delta z)$ & $10 \mathrm{~mm}$ \\
Number of slices & 3 \\
Slice gap & $\mathrm{NA}$ \\
Number of acquisitions $\left(N_{\mathrm{acq}}\right)$ & 1 \\
Read direction & Left-right \\
Slice locations & At isocenter, 3 orthogonal planes \\
Saturation pulses & None \\
RAM ${ }^{a}$ & $2 \mathrm{X}$ \\
Scan time & $6 \mathrm{sec}$ \\
${ }_{\text {ZZero padded from } 128 \text { by } 128 \text { points to } 256 \text { by } 256 \text { points. }}$
\end{tabular}

Infectious Diseases of the Brain

A4.2.3 
17. Let the patient know you are ready, and begin the scan.

Sequence 3: $T_{1}$-transverse head

18. Bring the sequence for a transverse $T_{1}$-weighted scan up onto the console. Set the imaging parameters as shown in Table A4.2.4.

19. Use the pilot sequence to set up the scan levels.

Table A4.2.3 Primary Clinical Imaging Parameters for Sequence 2: $T_{1}$-Sagittal Head

Patient position

Scan type

Imaging plane (orientation)

Central slice or volume center

Echo time $\left(T_{\mathrm{E}}\right)$

Repeat time $\left(T_{\mathrm{R}}\right)$

Flip angle (FA)

Fields of view $\left(\mathrm{FOV}_{\mathrm{x}}, \mathrm{FOV}_{\mathrm{y}}\right)$

Resolution $(\Delta x, \Delta y)$

Number of data points collected $\left(N_{\mathrm{x}}, N_{\mathrm{y}}\right)$

Display matrix $\left(D_{\mathrm{x}}, D_{\mathrm{y}}\right)$

Slice thickness $(\Delta z)$

Number of slices

Slice gap

Number of acquisitions $\left(N_{\text {acq }}\right)$

Read direction

Slice locations

Saturation pulses

Scan time
Supine

Spin echo

Sagittal

Midline head

$12 \mathrm{msec}$

$300 \mathrm{msec}$

$90^{\circ}$

$240 \mathrm{~mm}, 240 \mathrm{~mm}$

$1.25 \mathrm{~mm}, 0.94 \mathrm{~mm}$

192,256

256,256

$4 \mathrm{~mm}$

15

$1 \mathrm{~mm}$

1

Superior-inferior

Cover brain parenchyma

None

$1 \mathrm{~min}, 55 \mathrm{sec}$

Table A4.2.4 Primary Clinical Imaging Parameters for Sequence 3:

$T_{1}$-Transverse Head

Patient position

Scan type

Imaging plane (orientation)

Central slice or volume center

Echo time $\left(T_{\mathrm{E}}\right)$

Repeat time $\left(T_{\mathrm{R}}\right)$

Flip angle (FA)

Fields of view $\left(\mathrm{FOV}_{\mathrm{x}}, \mathrm{FOV}_{\mathrm{y}}\right)$

Resolution $(\Delta x, \Delta y)$

Number of data points collected $\left(N_{\mathrm{x}}, N_{\mathrm{y}}\right)$

Display matrix $\left(D_{\mathrm{x}}, D_{\mathrm{y}}\right)$

Slice thickness $(\Delta z)$

Number of slices

Slice gap

Number of acquisitions $\left(N_{\text {acq }}\right)$

Read direction

Slice locations

Saturation pulses

Scan time
Supine

Spin echo

Transverse (planum sphenoidale

line)

Mid-cranium

$12.1 \mathrm{msec}$

$500 \mathrm{msec}$

$90^{\circ}$

$240 \mathrm{~mm}, 180 \mathrm{~mm}$

$0.94 \mathrm{~mm}, 0.94 \mathrm{~mm}$

256, 192

256, 256

$5 \mathrm{~mm}$

24

$1 \mathrm{~mm}$

2

Anterior-posterior

Foramen magnum to vertex

None

2 min, $36 \mathrm{sec}$ 
20. Let the patient know you are ready, and begin the scan.

\section{Sequence 4: FLAIR transverse head}

21. Bring the sequence for a transverse FLAIR scan up onto the console. Set the imaging parameters as shown in Table A4.2.5.

22. Use the pilot sequence to set up the scan levels and a caudal saturation pulse.

23. Let the patient know you are ready, and begin the scan.

\section{Sequence 5: FSE transverse head}

24. Bring the sequence for a transverse FSE scan up onto the console. Set the imaging parameters as shown in Table A4.2.6.

25. Use the pilot sequence to set up the scan levels.

26. Let the patient know you are ready, and begin the scan.

\section{Sequence 6: $T_{1}$-transverse post-gadolinium head}

27. Bring the sequence for a transverse $T_{1}$-weighted scan up onto the console. Set the imaging parameters as shown in Table A4.2.7.

28. Use the pilot sequence to set up the scan levels.

29. Leaving the patient in the magnet, inject the contrast agent. This may be by either hand or mechanical injector. Observe the injection to insure there is no extravasation of the contrast agent. Flush the line with $10 \mathrm{ml}$ of sterile normal saline. The scan may begin as soon as the injection is completed. Alternatively, the scanning table may be advanced out of the magnet for the injection, but the patient must remain in place.

A dose of $0.1 \mathrm{mmol} / \mathrm{kg}$ of contrast agent is usually given.

Table A4.2.5 Primary Clinical Imaging Parameters for Sequence 4: FLAIR Transverse Head

\begin{tabular}{ll}
\hline Patient position & Supine \\
Scan type & FLAIR-FSE \\
Imaging plane (orientation) & Transverse (parallel to AC-PC line) \\
Central slice or volume center & Mid-cranium \\
Echo time $\left(T_{\mathrm{E}}\right)$ & $125 \mathrm{msec}$ (effective) \\
Echo train length (ETL) & Fixed (generally 12) \\
Repeat time $\left(T_{\mathrm{R}}\right)$ & $6000 \mathrm{msec}$ \\
Inversion time $\left(T_{\mathrm{I}}\right)$ & $1900 \mathrm{msec}$ \\
Flip angle $(\mathrm{FA})$ & $180^{\circ}$ \\
Fields of view $\left(\mathrm{FOV}, \mathrm{V}_{\mathrm{x}}\right.$, FOV $\left.\mathrm{y}\right)$ & $240 \mathrm{~mm}, 197 \mathrm{~mm}$ \\
Resolution $(\Delta x, \Delta y)$ & $0.94 \mathrm{~mm}, 0.97 \mathrm{~mm}$ \\
Number of data points collected $\left(N_{\mathrm{x}}, N_{\mathrm{y}}\right)$ & 256,204 \\
Display matrix $\left(D_{\mathrm{x}}, D_{\mathrm{y}}\right)$ & 256,256 \\
Slice thickness $(\Delta z)$ & $5 \mathrm{~mm}$ \\
Number of slices & 24 \\
Slice gap & $1 \mathrm{~mm}$ \\
Number of acquisitions $\left(N_{\mathrm{acq}}\right)$ & 1 \\
Read direction & Anterior-posterior \\
Slice locations & Foramen magnum to vertex \\
Saturation pulses & Caudal to saturate arterial flow \\
Scan time & 2 min, 48 sec \\
\hline
\end{tabular}

Infectious Diseases of the Brain 
Table A4.2.6 Primary Clinical Imaging Parameters for Sequence 5: FSE

Transverse Head

Patient position

Scan type

Imaging plane (orientation)

Central slice or volume center

Echo time $\left(T_{\mathrm{E}}\right)$

Echo train length (ETL)

Repeat time $\left(T_{\mathrm{R}}\right)$

Flip angle (FA)

Fields of view $\left(\mathrm{FOV}_{\mathrm{x}}, \mathrm{FOV}_{\mathrm{y}}\right)$

Resolution $(\Delta x, \Delta y)$

Number of data points collected $\left(N_{\mathrm{x}}, N_{\mathrm{y}}\right)$

Display matrix $\left(D_{\mathrm{x}}, D_{\mathrm{y}}\right)$

Slice thickness $(\Delta z)$

Number of slices

Slice gap

Number of acquisitions $\left(N_{\text {acq }}\right)$

Read direction

Slice locations

Saturation pulses

Scan time
Supine

Fast spin echo (FSE)

Transverse (planum sphenoidale

line)

Mid-cranium

$105 \mathrm{msec}$ (effective)

16

$5616 \mathrm{msec}$

$90^{\circ}$

$240 \mathrm{~mm}, 180 \mathrm{~mm}$

$0.63 \mathrm{~mm}, 0.70 \mathrm{~mm}$

384,256

384,384

$5 \mathrm{~mm}$

24

$1 \mathrm{~mm}$

2

Anterior-posterior

Foramen magnum to vertex

Caudal to saturate arterial flow

$3 \mathrm{~min}, 22 \mathrm{sec}$

Table A4.2.7 Primary Clinical Imaging Parameters for Sequence 6:

$T_{1}$-Transverse Post-Gadolinium Head

Patient position

Scan type

Imaging plane (orientation)

Central slice or volume center

Echo time $\left(T_{\mathrm{E}}\right)$

Repeat time $\left(T_{\mathrm{R}}\right)$

Flip angle (FA)

Fields of view $\left(\mathrm{FOV}_{\mathrm{x}}, \mathrm{FOV}_{\mathrm{y}}\right)$

Resolution $(\Delta x, \Delta y)$

Number of data points collected $\left(N_{\mathrm{x}}, N_{\mathrm{y}}\right)$

Display matrix $\left(D_{\mathrm{x}}, D_{\mathrm{y}}\right)$

Slice thickness $(\Delta z)$

Number of slices

Slice gap

Number of acquisitions $\left(N_{\text {acq }}\right)$

Read direction

Slice locations

Saturation pulses

Scan time

\section{Supine}

Spin echo

Transverse (planum sphenoidale

line)

Mid-cranium

$12.1 \mathrm{msec}$

$500 \mathrm{msec}$

$90^{\circ}$

$240 \mathrm{~mm}, 180 \mathrm{~mm}$

$0.94 \mathrm{~mm}, 0.94 \mathrm{~mm}$

256,192

256,256

$5 \mathrm{~mm}$

24

$1 \mathrm{~mm}$

2

Anterior-posterior

Foramen magnum to vertex

None

$2 \mathrm{~min}, 36 \mathrm{sec}$ 
Table A4.2.8 Primary Clinical Imaging Parameters for Sequence 7: $T_{1}$-Coronal Post-Gadolinium Head

\begin{tabular}{ll}
\hline Patient position & $\begin{array}{l}\text { Supine } \\
\text { Scan type }\end{array}$ \\
Imaging plane (orientation) & $\begin{array}{l}\text { Coronal (perpendicular to planum } \\
\text { sphenoidale line) }\end{array}$ \\
Central slice or volume center & Mid-cranium \\
Echo time $\left(T_{\mathrm{E}}\right)$ & $12.1 \mathrm{msec}$ \\
Repeat time $\left(T_{\mathrm{R}}\right)$ & $500 \mathrm{msec}$ \\
Flip angle $(\mathrm{FA})$ & $90^{\circ}$ \\
Fields of view $\left(\mathrm{FOV}, \mathrm{FOV}_{\mathrm{y}}\right)$ & $220 \mathrm{~mm}, 178 \mathrm{~mm}$ \\
Resolution $(\Delta x, \Delta y)$ & $0.86 \mathrm{~mm}, 0.93 \mathrm{~mm}$ \\
Number of data points collected $\left(N_{\mathrm{x}}, N_{\mathrm{y}}\right)$ & 256,192 \\
Display matrix $\left(D_{\mathrm{x}}, D_{\mathrm{y}}\right)$ & 256,256 \\
Slice thickness $(\Delta z)$ & $5 \mathrm{~mm}$ \\
Number of slices & 24 \\
Slice gap & $1 \mathrm{~mm}$ \\
Number of acquisitions $\left(N_{\mathrm{acq}}\right)$ & 2 \\
Read direction & Superior-inferior \\
Slice locations & Frontal to occipital cerebral poles \\
Saturation pulses & None \\
Scan time & $2 \mathrm{~min}, 36$ sec \\
\hline
\end{tabular}

This is essentially the same sequence as the pre-gadolinium scan, but can be performed with the addition of a flow compensation $(F C)$ pulse to better delineate the cerebral vessels, and a magnetization transfer (MT) pulse to optimize enhancing lesion detection.

\section{Sequence 7: $T_{1}$-coronal post-gadolinium head}

30. Bring the sequence for a $T_{1}$-weighted scan up onto the console. Set the imaging parameters as shown in Table A4.2.8.

31. Use the pilot sequence to set up the scan levels.

32. Let the patient know you are ready, and begin the scan.

Small abscesses may be much better delineated with a smaller field of view (FOV) (e.g., $F O V_{x}=200 \mathrm{~mm}, F_{y}=150 \mathrm{~mm}$ ) and higher resolution thinner slice thickness (e.g., 3 $\mathrm{mm})$. Please note that this modification should be performed with one acquisition, and limited to the area of abnormality to avoid prolonged scan times.

When scanning pediatric patients (under 24 months) modify the transverse and coronal sequences by placing the FOV at $200 \mathrm{~mm}$, and the slice thickness and slice gap at $4 \mathrm{~mm}$ and $1 \mathrm{~mm}$, respectively. For neonates, premature infants, and other extremely small patients an extremity coil may be used.

This sequence may be performed with the addition of a flow compensation (FC) pulse to better delineate the cerebral vessels, and a magnetization transfer (MT) pulse to optimize enhancing lesion detection.

\section{SPECIAL SITUATIONS}

The two sequences that follow (gradient echo and magnetic resonance angiogram or venogram) are presented here for consideration by the radiologist in those patients suspected of meningitis for whom the Basic Protocol has failed to elucidate a clearly defined abnormality. Patients with meningitis can suffer venous hemorrhage, which can be obvious and massive but is often subtle with microhemorrhage. The gradient echo

\section{ALTERNATE PROTOCOL}

Infectious Diseases of the Brain

A4.2.7 
sequence is the most sensitive MR sequence for detecting the magnetic susceptibility effect of the products of hemorrhage, particularly hemosiderin, and can display not only the helpful diagnostic clue of a hemorrhagic lesion but may well bring other lesions to the attention of the radiologist. This may in turn impact upon patient prognosis, or guide more effective follow-up imaging. Magnetic resonance angiography or venography can aid in detection of an associated (inflammatory) angiitis, most often manifest in the meningitis patient as venous thrombosis. Gadolinium administration may disclose such abnormalities more effectively, as it provides greater signal-to-noise within the vascular tree. This improves conspicuity of subtle findings in the larger vessels and increases visibility of the smaller vascular structures.

\section{Set up patient and equipment}

1. Use the same equipment and the same patient setup as for the previous method (see Basic Protocol, steps 1 to 11).

2. Run the pilot scan as sequence 1 in the Basic Protocol.

\section{Sequence 8: Coronal gradient echo head}

3. Bring the sequence for a gradient echo scan up onto the console. Set the imaging parameters as shown in Table A4.2.9.

4. Use the pilot sequence to set up the scan levels.

5. Let the patient know you are ready, and begin the scan.

\section{Sequence 9: Magnetic resonance angiography (MRA) and venography (MRV)}

6. Repeat step 29 in the Basic Protocol.

7. These sequences may be employed when the specific history provided for a patient suggests possible arterial or venous pathology, or if review of the spin-echo images indicates this possibility. In particular, the authors often find utility with MRV in evaluation of the meningitis patient. The reader is referred to the scanning parameters outlined for these sequences in Chapter A1.

Table A4.2.9 Primary Clinical Imaging Parameters for Sequence 8: Coronal Gradient Echo Head

\begin{tabular}{ll}
\hline Patient position & Supine \\
Scan type & 2D Gradient echo \\
Imaging plane (orientation) & Coronal \\
Central slice or volume center & Mid-cranium \\
Echo time $\left(T_{\mathrm{E}}\right)$ & $24.6 \mathrm{msec}$ \\
Repeat time $\left(T_{\mathrm{R}}\right)$ & $719 \mathrm{msec}$ \\
Flip angle $(\mathrm{FA})$ & $25^{\circ}$ \\
Fields of view $\left(\mathrm{FOV}_{\mathrm{x}}, \mathrm{FOV}_{\mathrm{y}}\right)$ & $220 \mathrm{~mm}, 220 \mathrm{~mm}$ \\
Resolution $(\Delta x, \Delta y)$ & $0.86 \mathrm{~mm}, 1.15 \mathrm{~mm}$ \\
Number of data points collected $\left(N_{\mathrm{x}}, N_{\mathrm{y}}\right)$ & 256,192 \\
Display matrix $\left(D_{\mathrm{x}}, D_{\mathrm{y}}\right)$ & 256,256 \\
Slice thickness $(\Delta z)$ & $5 \mathrm{~mm}$ \\
Number of slices & 21 \\
Slice gap & $1 \mathrm{~mm}$ \\
Number of acquisitions $\left(N_{\mathrm{acq}}\right)$ & 2 \\
Read direction & Superior-inferior \\
Slice locations & Frontal to occipital cerebral poles \\
Saturation pulses & None \\
Scan time & 4 min, 36 sec \\
\hline
\end{tabular}




\section{COMMENTARY}

\section{Background Information}

Meningitis may affect the dura mater (pachymeningitis), or more commonly the pia and arachnoid maters (leptomeningitis). The most common presentation is headaches, which may be accompanied by a stiff neck (meningismus), confusion, and disorientation. Fever is often present and cranial neuropathies may develop (Hansman-Whiteman et al., 1996; Taveras and Pile-Spellman, 1996; Wong and Quint, 1999). The disease may be viral, bacterial, or fungal in origin. The most common etiology, and the most difficult form to diagnose is viral meningitis as imaging studies are usually negative and cerebralspinal fluid (CSF) abnormalities are not specific. Viral cultures may reveal the pathogen as mumps or enterovirus (most commonly), and occasionally the herpes or human immunodeficiency viruses. Bacterial disease is most often caused by E. coli in infants, $H$. influenza in children and in adults is predominantly secondary to meningococcal or pneumococcal species. Tuberculous and parasitic meningitides occur in endemic populations. Fungal meningitis is mostly found in the immunocompromised population with many pathogens described, to include cryptococcus, coccidioidomycosis, and aspergillus (Harris and Enterline, 1997). The basilar meninges are most commonly affected by the tuberculosis and fungal pathogens, a condition referred to as basilar meningitis. Alternate etiologies for basilar meningitis are other granulomatous conditions, including sarcoid. Basilar meningitis is the most common form for chronic meningitis, though it may also present acutely.

The route of infection is usually hematogenous for leptomeningitis, primarily to the choroid plexus and then the CSF. But contiguous spread of disease may be seen, particularly in patients with sinus or middle ear disease, or post-operative or traumatized patients. However, all patients with meningitis should be carefully evaluated for the primary source of the infection.

Leptomeningitis begins in the pia mater, a vascular structure that allows for the passage of leukocytes into the subarachnoid space where the relatively avascular arachnoid membrane traps the cells, and pus accumulates. A phlebitis eventually ensues in the traversing cortical veins that leads to thrombosis, cortical venous congestion, and abscess and/or small infarctions. With subarachnoid space involve- ment, the ependyma is at risk and ependymitis may ensue, particularly in neonatal disease. Obstruction to normal CSF drainage pathways is the norm because of loculations that may form in the subarachnoid or ventricular spaces, or sludging of inflammatory debris within these pathways or the arachnoid granulations. This may result in hydrocephalus and increased intracranial pressure, as well as sterile sympathetic subdural effusions. These latter fluid collections are most often noted in children with $H$. influenza infection along the frontal and parietal convexities, and may turn into subdural empyema.

While leptomeningitis is a medical emergency, a subdural empyema is a neurosurgical emergency as these infections can progress quite rapidly to vascular thrombosis of many cortical veins or larger dural venous sinuses. This can produce large cerebral venous infarctions and hemorrhage. These empyemas are usually the result of sinusitis (predominantly frontal), though they can occur secondary to otitis media, and trauma.

The only significant differential diagnostic considerations in patients with meningitis are meningeal carcinomatosis, noninfectious granulomatous meningitis (i.e., sarcoid), posttraumatic meningeal effusions such as may be seen in child abuse, and benign subdural hygromas of infancy. The clinical scenario is usually all that is necessary to differentiate among these entities. More important than differentiation from these other conditions, is the initial detection of the often subtle findings of the disease and then proper ascription of an infectious agent, and all of this in a timely manner in these often gravely ill patients.

\section{Critical Parameters and Troubleshooting}

There are several parameters that enhance the sensitivity and utility of the Basic Protocol. The reader is referred to the prior unit on cerebral abscess (UNIT A4.I) for further discussion of several key parameters to optimize when imaging the patient suspected of meningitis. Specifically, as the early findings of meningitis may be quite subtle (or not yet present) proper attention to these parameters may make the difference between a positive and negative study. These include the proper selection of the $T_{\mathrm{I}}$ (inversion time) for the FLAIR images to effect nulling of hyperintense CSF signal and produce dark CSF. This enables enhanced detection of
Infectious

Diseases of the Brain

A4.2.9 
abnormal periventricular high $T_{2}$-signal intensity for the important determination of ventriculitis/ependymitis, hydrocephalus with transependymal flow of CSF, as well as better evaluation of the subarachnoid space. Additionally, the optional use of the MT pulse for the post-gadolinium sequences enhances the sensitivity for early detection of subtle foci of abnormal enhancement. However, it is the authors' experience that obtaining post-gadolinium sequences in multiple planes is also essential to not only aid in the detection of meningitis, but to confirm subtle findings seen in another plane. The authors recommend both transverse and coronal planes as these offer the best evaluation of the subdural and subarachnoid spaces, as well as the ependymal surfaces of the ventricles for the ependymal and subependymal enhancement of ventriculitis. This may be difficult or impossible to demonstrate in a single (transverse) plane. A double dose of gadolinium contrast to better demonstrate the abnormal meningeal enhancement is rarely used.

When considering the optional sequences, the optimal choice of $T_{\mathrm{E}}$ on the gradient echo sequence (sequence 8 ) is important to allow for detection of foci of microhemorrhage. A longer $T_{\mathrm{E}}$ will demonstrate increased $T_{2}$-contrast, and magnetic susceptibility with more pronounced low signal "blooming" from foci of hemorrhage containing hemosiderin (though at the expense of overall tissue signal to noise ratio). Generally, $T_{\mathrm{E}}$ values of $<25 \mathrm{msec}$ are less sensitive for this purpose (NOTE: the authors use a $T_{\mathrm{E}}$ of $24.6 \mathrm{msec}$ ). For similar reasons, this "blooming" makes for poor evaluation of signal at the skull-brain interface, particularly at the skull base. Thus, the meninges are not well demonstrated on the gradient echo sequence.

The MR angiographic sequences (MRA and MRV) are utilized for large vessel status rather than subtle small vessel findings. The latter are much better evaluated on catheter angiograms, and are generally not present unless there are marked abnormalities noted on spin echo sequences (Pomper et al., 1999). Thus, MRA is used to evaluate the circle-of-Willis, while MRV is used for the larger dural venous sinuses. The authors routinely employ MRV in patients suspected of meningitis as these sinuses are commonly affected by the disease (Fig. A4.2.1), but will only obtain an MRA if there is supportive evidence of arterial vascular pathology on spin-echo sequences, such as suspicious hemorrhage or abnormal vascular contours or luminal signal.

\section{Imaging of meningitis by magnetic resonance diffusion}

The Basic Protocol outlined above is quite sufficient in most instances of meningitis imaging and represents the standard for imaging of the patient suspected for meningitis. dMRI has no defined role in the diagnosis of meningitis but can be utilized in the patient suspected of meningitis to assess for one of the more common complications of the disease, specifically cerebral infarction. These are usually venous infarctions and may often be accompanied by hemorrhage, especially when larger. Please refer to the scan parameters and set-up outlined in detail in UNITA4.I of this chapter for performance of this sequence.

\section{Anticipated Results}

The role of MR imaging in the evaluation of the patient suspected to have meningitis is predominantly to assess for the presence of abscess, localized empyema and ventriculitis. The paranasal sinuses and temporal bones may also be scrutinized, but are mostly better seen with computed tomography (CT). The other main benefit of MR is to assess for the complications of the disease such as hydrocephalus, cerebral infarction, and to evaluate treatment efficacy and disease progression. However, it must always be kept in mind and readily communicated to referring clinicians, that a negative imaging result in a patient suspected of meningitis must always be followed by a diagnostic CSF tap. This test remains the "gold standard" and has higher sensitivity and specificity for meningitis than does MRI, as well as the added necessary benefit of allowing for specific pathogen identification and culture, and determination of antibiotic sensitivities.

At initial presentation, meningitis typically appears on MR imaging as either a thickening of the meningeal layer(s), or as a collection of fluid signal material in either the subarachnoid or subdural compartments. The disease may be localized or diffuse, and may only involve the basilar meninges. Depending on the pathogen and the burden of disease these findings may be either quite subtle or striking. If the disease resides in the subdural compartment (pachymeningitis), then the dura may appear abnormally thick and low in signal intensity on $T_{1}$-weighted images, while $T_{2}$-weighting will disclose high fluid-like signal. The signal of an empyema is between that of CSF and brain on $T_{1}$-weighted imaging and occasionally, a fluiddebris level may be demonstrated in the subdural space. Gadolinium sequences will dis- 
A

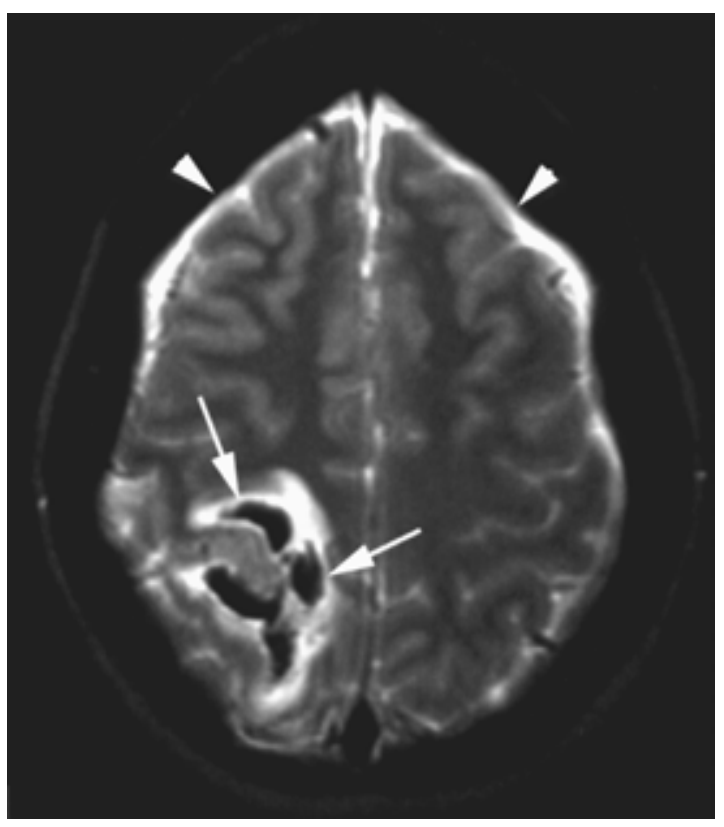

B

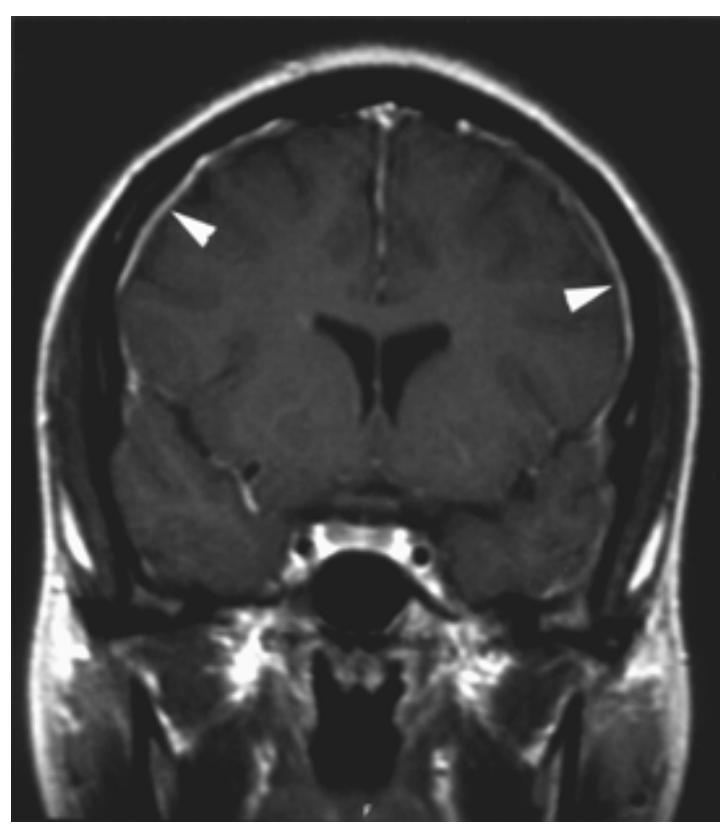

C

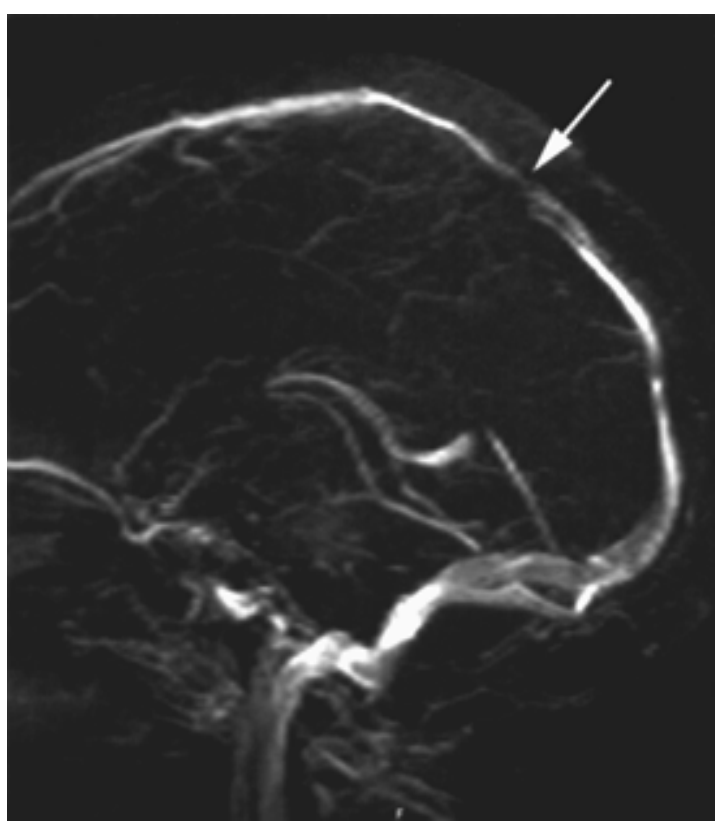

Figure A4.2.1 This 20-year-old female with known pulmonary tuberculosis presented with new-onset seizures. MRI shows parenchymal hemorrhage of a venous infarction pattern on this $T_{2}$-weighted $\left(T_{\mathrm{R}}=2500 \mathrm{msec}, T_{\mathrm{E}}=\right.$ $80 \mathrm{msec}, N_{\mathrm{acq}}=0.75$ ) transverse $(\mathbf{A})$ vertex image (arrows). Also noted is abnormal increased $T_{2}$ signal intensity suggesting dural thickening in bifrontal distribution (arrowheads). Enhancement of the dura confirming pachymeningitis is seen on the $T_{1}$-weighted $\left(T_{\mathrm{R}}=600 \mathrm{msec}, T_{\mathrm{E}}=23 \mathrm{msec}, N_{\text {acq }}=2\right)$ post-contrast coronal (B) image (arrowheads). The MRV (C) discloses abnormal attenuation of the superior sagittal sinus (arrow) in parietal location, confirming a stenosis likely causing the venous hypertension and infarction in this patient. 


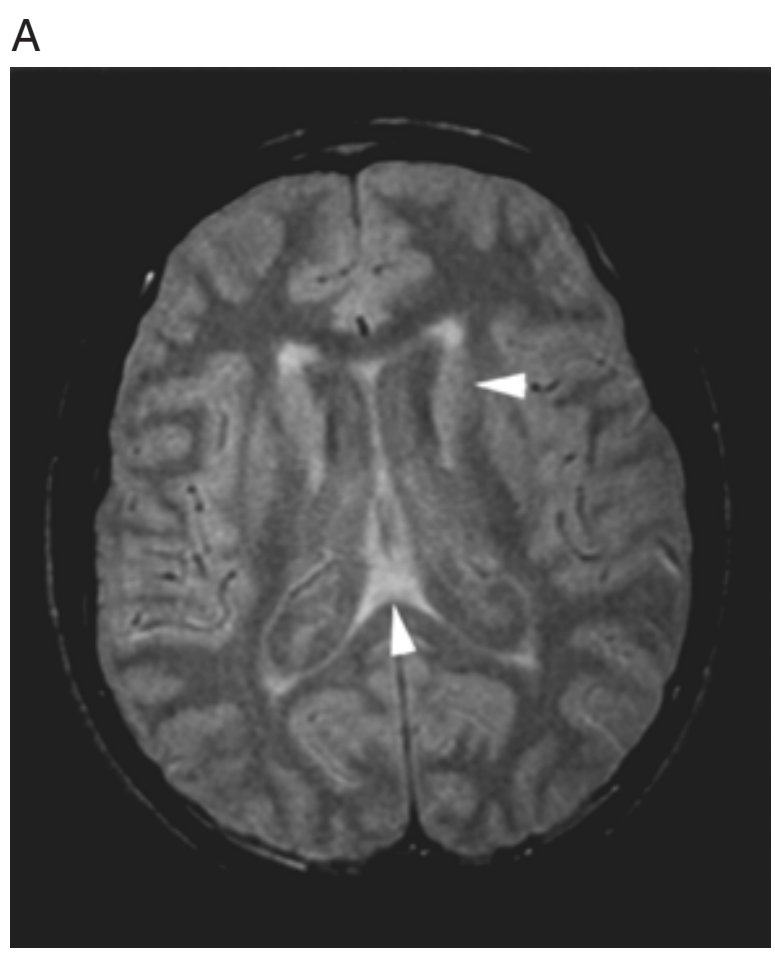

B

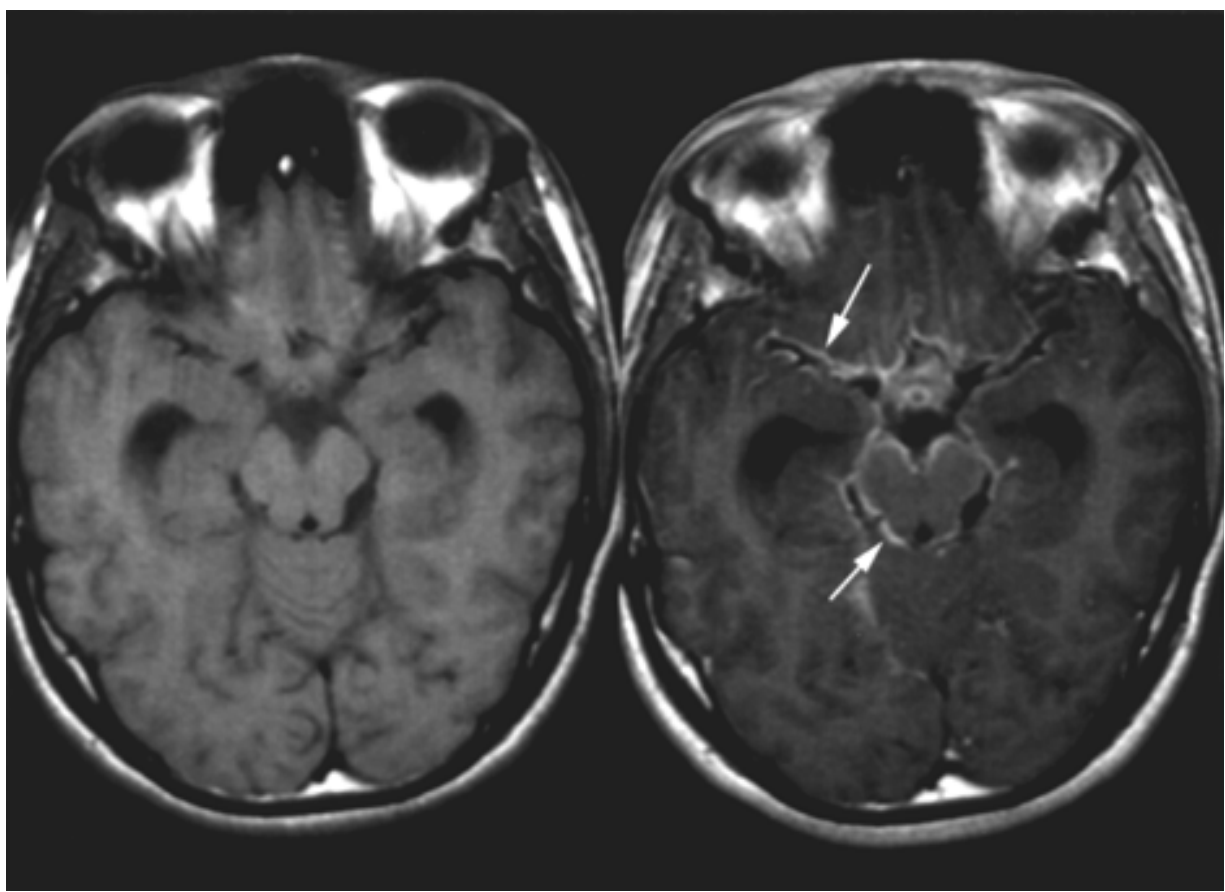

Figure A4.2.2 This 16-year-old boy presented to the emergency room with subtle mental status changes and a fever of one week duration. A proton-density weighted ( $T_{\mathrm{R}}=2500 \mathrm{msec}, T_{\mathrm{E}}=30$ msec, $\left.N_{\text {acq }}=0.75\right)$ transverse image $(A)$ through the lateral ventricles shows increased ventricular volume relative to the extra-transverse CSF spaces. There is also subtle abnormal increased signal seen rimming the ventricles (arrowheads) compatible with acute hydrocephalus and transependymal flow of CSF. The transverse pre- and post-contrast $\left(T_{\mathrm{R}}=500 \mathrm{msec}, T_{\mathrm{E}}=11 \mathrm{msec}, N_{\mathrm{acq}}=2\right)$ images (B) at the level of the basilar cisterns demonstrates diffuse "sugar-coating" enhancement of the basilar meninges (arrows on the post-contrast image) diagnostic of basilar meningitis. A positive tuberculin skin test was obtained and CSF analysis was confirmatory for tuberculosis.

Meningitis

A4.2.12 
close abnormal thickening and prominent enhancement of the involved dura. A contiguous infectious source for the collection should be sought, with prime candidates being the middle ear spaces and the frontal sinuses. The gradient echo and MRV sequences may have utility in these patients, disclosing hemorrhagic foci (these may be numerous) and dural sinus thrombosis (especially the superior sagittal, straight, and transverse sinuses), respectively.

With leptomeningitis, abnormal fluid-like signal may be found within the subarachnoid spaces with abnormally prominent or asymmetric sulci seen. Similar findings may be found in the basilar compartment and the spaces of Virchow-Robin that may be subtly involved with an unusual prominence and number to the spaces noted. Gelatinous pseudocysts have been described with a "soap-bubble" appearance within the basilar Virchow-Robin spaces in patients with cryptococcal meningitis. These lesions appear similar in signal to CSF, but are delineated by a barely discernible membrane. Gadolinium images will show enhancement of the basilar cisterns, the perivascular Virchow-Robin spaces, or a "sugar-coating" of the brain and brainstem in the involved regions (Fig. A4.2.2). The enhancing leptomeninges can appear thickened and even nodular, though this is less common. This is the inflamed pia mater with abnormal thickening and enhancement. Inflammatory septa may be found within the subarachnoid space or within the ventricles, and ependymal enhancement may be present, possibly with periventricular edema. Cortical hyperintensity on $T_{2}$-weighted images may be noted from edema related to adjacent meningeal inflammation and associated cerebritis or abscess may be seen. Hydrocephalus is a common occurrence eventually in the meningitis patient and should be sought. Additionally, leptomeningitis may rarely lead to vasculitic involvement of the major arterial tributaries of the brain as they traverse the affected subarachnoid space. This may manifest as subarachnoid or intraventricular hemorrhage due to rupture of the involved vessel, which can become aneurysmal as a result of the inflammation. These may be true or false (pseudo-) aneurysms. Alternatively, infarction of perfused areas of the brain may result.

Chronically, bulky subarachnoid exudates may result, especially with basilar meningitis. Abnormal enhancement may be seen with MRI even years after disease onset, and atrophy may result. Meningeal calcifications can occur, especially with tuberculosis.
An unusual form of meningitis (though exceedingly common in endemic regions) is cysticercal meningitis. This disease also commonly causes parenchymal infection and has different appearances depending on when in the infectious cycle it is imaged (Hansman-Whiteman et al., 1996; Taveras and Pile-Spellman, 1996; Wong and Quint, 1999). This cycle runs between 2 and 10 years for the pork tapeworm larva ( $T$. solium), averaging 5 years. It usually demonstrates one or several gray-white matter junction or subarachnoid/ventricular rounded lesions measuring 1 to $2 \mathrm{~mm}$ in size and harboring a small mural nodule, or scolex. This is the dead parasite, as there is generally no inflammatory involvement of the central nervous system (CNS) until the parasite dies. These lesions eventually calcify and may reveal inert imaging findings of isointense signal to normal brain on $T_{1}$-weighted images with isointense to hypointense $T_{2}$ signal. Variable edema and wall enhancement are noted until the chronic stage, however.

\section{Literature Cited}

Hansman-Whiteman, M., Bowen, B.C., DonovanPost, M.J., and Bell, M.D. 1996. Intracranial infection. In Magnetic Resonance Imaging of the Brain and Spine, 2nd edition (Atlas, S.W., ed.) pp. 707-772. Lippincott-Raven, Philadelphia.

Harris, D.E. and Enterline, D.S. 1997. Neuroimaging of AIDS. I. Fungal infections of the central nervous system. Neuroimaging Clin. N. Am. 7:187-198.

Pomper, M.G., Miller, T.J., Stone, J.H., Tidmore, W.C., and Hellmann, D.B. 1999. CNS vasculitis in autoimmune disease: MR imaging findings and correlation with angiography. A.J.N.R. 20:75-85.

Shellock, F.G. 1996. Pocket Guide to MR Procedures and Metallic Objects. Lippincott-Raven, Philadelphia.

Taveras, J.M. and Pile-Spellman, J. 1996. Inflammatory diseases. In Neuroradiology, 3rd ed. pp. 259-326. Williams and Wilkins, Baltimore.

Wong, J. and Quint, D.J. 1999. Imaging of central nervous system infections. Semin. Roentgenol. 34:123-143.

\section{Key Reference}

Harris, D.E. and Enterline, D.S. 1997. See above.

Contains lucid explanations for the physics and basic scan parameters of standard and advanced magnetic resonance imaging studies.

Contributed by Andrew E. Auber and Clifford Belden

Brooke Army Medical Center

San Antonio, Texas
Infectious

Diseases of the Brain

A4.2.13 